\title{
The Smith Normal Form of a Partitioned Matrix
}

\author{
Morris Newman \\ Institute for Basic Standards, National Bureau of Standards, Washington, D.C. 20234
}

(September 11, 1973)

It is shown that if

$$
M=\left[\begin{array}{llll}
M_{11} & M_{12} & \ldots & M_{1 t} \\
0 & M_{22} & \ldots & M_{2 t} \\
0 & 0 & \ldots & M_{t t}
\end{array}\right]
$$

is a matrix over a principal ideal ring $R$ such that the matrices $M_{i i}$ are square and have pairwise relatively prime determinants, then the Smith normal form of $M$ is the same as the Smith normal form of $M_{11} \dot{+} M_{22} \dot{+} \ldots \dot{+} M_{t t}$

Key words: Elementary divisors; invariant factors; partitioned matrices; Smith normal form.

Let $R$ be a principal ideal ring. Let $A$ be an $r \times r$ matrix over $R, B$ an $s \times s$ matrix over $R$. It is well known that the elementary divisors of $A+B$ are the elementary divisors of $A$ together with the elementary divisors of $B$, which allows us to reconstruct the Smith Normal Form (hereafter abbreviated S.N.F.) of $A \dot{+} B$ from the invariant factors of $A$ and of $B$ (see [1], ${ }^{1}$ for example). There is a noteworthy instance which merits special attention: namely, when the determinants of $A$ and $B$ are relatively prime. This note is devoted to this case.

We let $S(M)$ denote the S.N.F. of any matrix $M$ over $R$, and $I_{n}$ denote the identity matrix of order $n$. $I$ will denote an identity matrix of unspecified order.

We first prove

Theorem 1: Suppose that $(\operatorname{det}(\mathrm{A}), \operatorname{det}(\mathrm{B}))=1$, and that

$$
\begin{aligned}
& \mathrm{S}(\mathrm{A})=\operatorname{diag}\left(\alpha_{1}, \alpha_{2}, \ldots, \alpha_{\mathrm{r}}\right), \\
& \mathrm{S}(\mathrm{B})=\operatorname{diag}\left(\beta_{1}, \beta_{2}, \ldots, \beta_{\mathrm{s}}\right),
\end{aligned}
$$

so that $\alpha_{1}, \alpha_{2}, \ldots, \alpha_{\mathrm{r}}$ are the invariant factors of $\mathrm{A}, \beta_{1}, \beta_{2}, \ldots, \beta_{\mathrm{s}}$ the invariant factors of $\mathrm{B}$; and assume for definiteness that $\mathrm{r} \leqslant \mathrm{s}$. Then

(1) $\mathrm{S}(\mathrm{A} \dot{+} \mathrm{B})=\mathrm{I}_{\mathrm{r}} \dot{+} \operatorname{diag}\left(\beta_{1}, \beta_{2}, \ldots, \beta_{\mathrm{s}-\mathrm{r}}\right) \dot{+} \operatorname{diag}\left(\alpha_{1} \beta_{\mathrm{s}-\mathrm{r}+1}, \alpha_{2} \beta_{\mathrm{s}-\mathrm{r}+2}, \ldots, \alpha_{\mathrm{s}} \beta_{\mathrm{r}}\right)$.

AMS Subject Classification: 1530, 1545, 1548.

${ }^{1}$ Figures in brackets indicate the literature references at the end of this paper. 
Proof: A moment's consideration shows that the expression given in (1) for $S(A \dot{+} B)$ is just $S\left(I_{s} \dot{+} A\right) S\left(I_{r} \dot{+} B\right)$. Now

$$
\begin{aligned}
A \dot{+} B & =\left[\begin{array}{cc}
A & 0 \\
0 & B
\end{array}\right]=\left[\begin{array}{cc}
A & 0 \\
0 & I_{s}
\end{array}\right]\left[\begin{array}{ll}
I_{r} & 0 \\
0 & B
\end{array}\right] \\
& =\left(A+I_{s}\right)\left(I_{r}+B\right) .
\end{aligned}
$$

But $A+I_{s}$ and $I_{r} \dot{+} B$ have relatively prime determinants, and it is known that if $M, N$ are matrices over $R$ of the same size such that $(\operatorname{det}(M), \operatorname{det}(N))=1$, then $S(M N)=S(M) S(N)$ (see [2]). It follows that

$$
\begin{aligned}
S(A+B) & =S\left(A+I_{s}\right) S\left(I_{r} \dot{+} B\right) \\
& =S\left(I_{s} \dot{+} A\right) S\left(I_{r}+B\right) .
\end{aligned}
$$

This concludes the proof.

Now let $T$ be any $r \times s$ matrix over $R$. Then provided that $(\operatorname{det}(A), \operatorname{det}(B))=1$, the next result shows that $T$ plays no part in determining the S.N.F. of

$$
\left[\begin{array}{ll}
A & T \\
0 & B
\end{array}\right]
$$

Specifically, we prove

THEOREM 2: Suppose that $(\operatorname{det}(\mathrm{A}), \operatorname{det}(\mathrm{B}))=1$. Then the S.N.F. of

$$
\left[\begin{array}{ll}
\mathrm{A} & \mathrm{T} \\
0 & \mathrm{~B}
\end{array}\right]
$$

is the same as the S.N.F. of

$$
\left[\begin{array}{cc}
\mathrm{A} & 0 \\
0 & \mathrm{~B}
\end{array}\right]
$$

Proof: Let $A^{\text {adj }}$ be the adjoint of $A, B^{\text {adj }}$ the adjoint of $B$, so that $A^{\text {adj }}, B^{\text {adj }}$ are matrices over $R$ satisfying

$$
\begin{aligned}
& A A^{\text {adj }}=A^{\text {adj }} A=\operatorname{det}(A) \cdot I_{r}, \\
& B B^{\text {adj }}=B^{\text {adj }} B=\operatorname{det}(B) \cdot I_{s} .
\end{aligned}
$$

Since $(\operatorname{det}(A), \operatorname{det}(B))=1$, elements $\alpha, \beta$ of $R$ exist such that

$$
\alpha \operatorname{det}(A)+\beta \operatorname{det}(B)=1 .
$$

Now consider the equation

$$
\left[\begin{array}{cc}
I_{r} & X \\
0 & I_{s}
\end{array}\right]\left[\begin{array}{ll}
A & 0 \\
0 & B
\end{array}\right]\left[\begin{array}{ll}
I_{r} & Y \\
0 & I_{s}
\end{array}\right]=\left[\begin{array}{ll}
A & T \\
0 & B
\end{array}\right] .
$$


Then (2) holds if and only if

$$
T=A Y+X B
$$

But (3) may be satisfied by choosing

$$
X=\beta T B^{\text {adj }}, Y=\alpha A^{\text {adj }} T \text {. }
$$

Thus (2) has a solution in matrices $X, Y$ over $R$, and it follows that $\left[\begin{array}{rr}A & T \\ 0 & B\end{array}\right]$ and $\left[\begin{array}{rr}A & 0 \\ 0 & B\end{array}\right]$ are equivalent, and hence have the same S.N.F. This concludes the proof.

We remark that because of Theorem 1, the S.N.F. of $\left[\begin{array}{ll}A & T \\ 0 & B\end{array}\right]$ is completely determined by the invariant factors of $A$ and the invariant factors of $B$, when $(\operatorname{det}(A), \operatorname{det}(B))=1$.

This result is definitely false if the determinant condition is removed. For example, the S.N.F. of $\left[\begin{array}{ll}4 & 0 \\ 0 & 6\end{array}\right]$ is $\left[\begin{array}{ll}2 & 0 \\ 0 & 12\end{array}\right]$, but the S.N.F. of $\left[\begin{array}{ll}4 & 1 \\ 0 & 6\end{array}\right]$ is $\left[\begin{array}{rr}1 & 0 \\ 0 & 24\end{array}\right]$.

We note in passing that if

$$
S(A)=\operatorname{diag}\left(\alpha_{1}, \alpha_{2}, \ldots, \alpha_{r}\right),
$$

then

$$
S(A \dot{+} A \dot{+} \ldots \dot{+} A)=\alpha_{1} I_{k} \dot{+} \alpha_{2} I_{k} \dot{+} \ldots \dot{+} \alpha_{r} I_{k},
$$

where there are $k$ replicas of $A$ in the direct sum.

Theorem 2 may be generalized as follows:

THEOREM 3: Let $\mathrm{M}$ be a matrix over $\mathrm{R}$, and suppose that $\mathrm{M}$ may be partitioned as

$$
\mathbf{M}=\left[\begin{array}{cccc}
M_{11} & M_{12} & \ldots & M_{1 t} \\
0 & M_{22} & \ldots & M_{2 t} \\
& \ldots & & \\
0 & 0 & \ldots & M_{t t}
\end{array}\right]
$$

where the matrices $\mathrm{M}_{\mathrm{ii}}$ are square and have pairwise relatively prime determinants. Then the S.N.F. of $\mathrm{M}$ is determined by the invariant factors of the $\mathrm{M}_{\mathrm{ii}}$;

Proof: Put

$$
\mathrm{S}(\mathrm{M})=\mathrm{S}\left(\mathrm{M}_{11} \dot{+} \mathrm{M}_{22} \dot{+} \ldots \dot{+} \mathrm{M}_{\mathrm{tt}}\right) \text {. }
$$

$$
\begin{aligned}
A & =M_{11}, \\
T & =\left[M_{12}, \ldots, M_{1 t}\right], \\
B & =\left[\begin{array}{lll}
M_{22} & \ldots & M_{2 t} \\
0 & \ldots & \\
0 & \ldots & M_{t t}
\end{array}\right] .
\end{aligned}
$$

Then

$$
M=\left[\begin{array}{cc}
A & T \\
0 & B
\end{array}\right],
$$


and $(\operatorname{det}(A), \operatorname{det}(B))=1$. By Theorem 2 and the result on the multiplicativity of the S.N.F.,

$$
\begin{aligned}
S(M) & =S\left(\left[\begin{array}{ll}
A & 0 \\
0 & B
\end{array}\right]\right) \\
& =S\left(\left[\begin{array}{ll}
A & 0 \\
0 & I
\end{array}\right]\right) S\left(\left[\begin{array}{ll}
I & 0 \\
0 & B
\end{array}\right]\right),
\end{aligned}
$$

since $(\operatorname{det}(A), \operatorname{det}(B))=1$. Repeating this procedure with the matrix $B$, we ultimately obtain

$$
\begin{aligned}
S(M) & =S\left(M_{11} \dot{+} I\right) S\left(I \dot{+} M_{22} \dot{+} I\right) \ldots S\left(I \dot{+} M_{t t}\right) \\
& =S\left(M_{11} \dot{+} M_{22} \dot{+} \ldots \dot{+} M_{t t}\right) .
\end{aligned}
$$

This concludes the proof.

\section{References}

[1] Newman, Morris, Integral Matrices, Academic Press, New York (1972).

[2] - , On the Smith normal form, J. Res. Nat. Bur. Stand. (U.S.), 75B (Math. Sci.), Nos. 1\&2, 81-84 (Jan.-June 1971).

(Paper 78B1-392) 